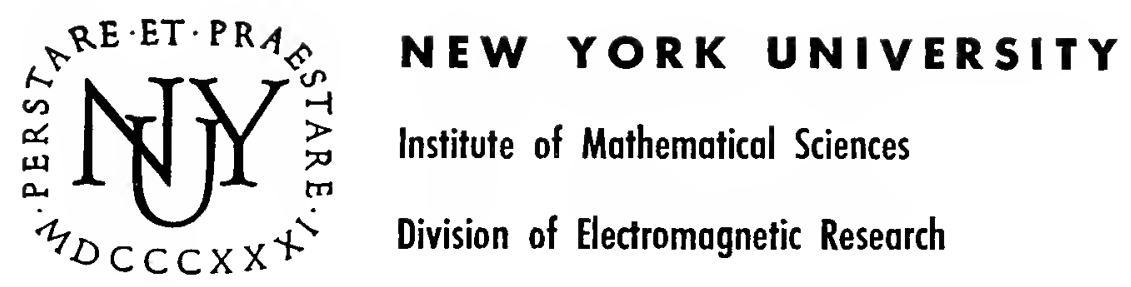

RESEARCH REPORT No. CX-55

\title{
Bounds on Elements of the S Matrix for Elastic Scattering: One Dimensional Scattering
}

RALPH BARTRAM and LARRY SPRUCH

\author{
Contract Nos. Af $19(60414555$ \\ DA 30-069-ORD-2581 \\ Nonr-285(49), NR 012-109
}

JUNE, 1961 



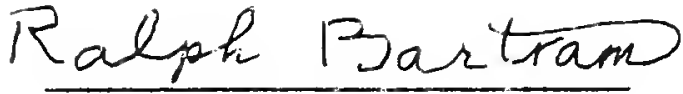

Ralph Bartram

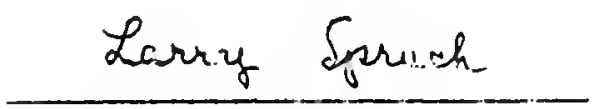

Larry Sprucl.

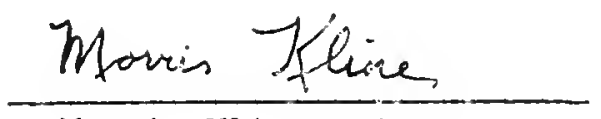

Morris Kline, Director

June, 1961
The research reported in this paper has been jointly sponsored by the Geophysics Research Directorate of the Air Force Cambridge Research Center, Air Research and Development Command, under Contract No. AF 19(604)4555, and the office of Ordnance Research under Contract No. DA-30-069-ORD-2581, Project No. 2360, and the Office of Naval Research under Contract Nonr-285(49), IVR 012-109. Reproduction in whole or in part is permitted for any purpose of the United States Government. 
Requests for additional copies by Agencies of the Department of Defense, their contractors, and other Government agencies should be directed to the:

ARMED SERVICES TECHNICAL INFORMATION AGENCY DOCUMENTS SERVICE CENTER

ARLINGTON HALL STATION

ARLINGTON I2, VIRGINIA

Department of Defense contractors must be established for ASTIA services or have their 'need-to-know' certified by the cognizant military agency of their project or contract. All other persons and organizations should apply to the:

U.S. DEPARTMENT OF COMMERCE OFFICE OF TECHNICAL SERVICES WASHLNGTON 25, D.C. 
2. Matrix formulation of the variational principle

3. Rigorous bounds on the error term 11

4. Bounds on $\alpha_{\theta}$ and on $\beta_{\theta} \quad 17$

5. Method of obtaining bounds 21

6. Numerical examples 24

References 30 



\section{INIRODUCTION}

Kato $^{1}$ has demonstrated the possibility of determining upper and lower bounds on cot $\eta$, where $\eta$ is the phase shift for arbitrary angular momentum for the quantum mechanical scattering of a particle by a static central potential. The basic interest of the method derives from the fact that the expression for either bound includes a variational trial function, and gives an error which is of second order in the difference between the trial function and the exact function. The bounds are therefore also variational estimates, and might be termed variational bounds. In subsequent studies, $2,3,4$ the case of angular momenta greater than zero was considered more intensively, some slight extensions and improvements in the formalism were effected, and some preliminary results vere obtained for the applicability of the method to scattering by compound systems with emphasis on the low energy scattering of positrons by Hydrogen atoms.

Static central potential scattering problems involve an infinite number of channels which are however uncoupled, a consequence of conservation of angular momentum. In other words, an expansion into partial waves leads to a diagonalization of the scattering matrix $\underset{\mathrm{m}}{\mathrm{S}}$ before the detailed analysis is begun. Furthermore, each angular momentum channel vave function is completely characterized by one real number, the real phase shift for that angular momentum. More generally, the Kato method can be applied whenever the S matrix can be diagonalized on some a priort basis (generally this will be through symmetry considerations); the resultant eigenmodes can then each be characterized by one 
real number. Examples ${ }^{5}$ include the one dimensional scattering problem for an even potential, $V(x)=V(-x)$, and the problem of electromagnetic waves in a rectangular wave guide which has an obstacle symmetric with respect to some plane perpendicular to the axis of symmetry. The latter case involves vector wave functions of three independent variables rather than scalar wave functions of one independent variable, but this causes no essential changes. In both cases, the eigenmodes are the even and odd standing wave solutions.

In this paper, we will consider multi-channel scattering in which the scattering matrix cannot be diagonalized a priori. More formally, we are concerned with elastic multi-channel scattering processes. We shall cast the Schroedinger equation into a form in which the potential is a real symmetric $N \times N$ matrix and the wave function is an $N \times I$ matrix, though it is not necessary to do so. The equation for neutron proton scattering with tensor forces is conveniently written in matrix form 6 with $N=2$, and we shall see in Section 2 that the same is true for the problem of one-dimensional scattering. It is our purpose to determine upper and lower bounds on the elements of the $\underset{\mathrm{S}}{\mathrm{S}} \operatorname{matrix}$, the $\mathrm{N} \times \mathrm{N}$ matrix which relates the $N$ outgoing waves to the $N$ incoming waves. The elements of $\underset{\mathrm{m}}{\mathrm{S}}$ are in general complex, so that there are $2 \mathrm{~N}^{2}$ real quantities in all, but it is well known that only $\frac{1}{2} N(N+I)$ of these real quantities are independent. 7

The procedure, in analogy with that of Kato, is to derive an integral variational principle which is in fact an identity and then to bound the explicitly exhibited second order term. Upper and lower bounds 
are first obtained on the elements $B_{i j}$ of $B$, where the susceptance matrix $\underset{m}{B}$ is the Inverse of the Cayley transform of $\mathrm{S}$. One can then also obtain bounds on the elements of $\mathrm{S}$, or on still another set of $\frac{1}{2} N(N+I)$ quantities which characterize the scattering, the $\mathrm{N}$ eigenphase shifts and the $\frac{1}{2} \mathrm{~N}(\mathrm{~N}-1)$ mixing parameters.

Numerical examples are calculated in section 6 for a one-dimensional attractive square well asymmetric with respect to the origin.

Because of the enoimous advantages of variational bounds over varlational estimates, it is of great interest to study the limits to which the Kato method can be extended. There are a number of obvious directions In which to proceed. We will list some systems of interest, starting with those already considered.

(1) Systems which Involve the scattering of a particle by a static central potential. We are then of course concermed with one channel processes.

(2) Systems which for sufficiently low energy of the incident particle involve, as does (I), only one (elastic) channel, or a priori separable (elastic) channels, but which are compound. We w1ll refer to a system as compound if it has spatial (as opposed to finite dimensional spin) internal degrees of freedom.

(3) Systems which involve the scattering of a particle by a potential which is static (the scattering system has no spatial degrees of freedom) and which can be represented by a hermitian $\mathrm{N} \times \mathrm{N}$ matrix. It Is assumed that the $N>1$ elastic channels cannot be uncoupled in any a priori way. 
(4) Systems which are compound and which involve $\mathbb{N}>I$ elastic channels.

(5) Systems which involve inelastic scattering.

From a purely formal point of view, case (2) is included in the original paper of Kato. I Unfortunately, the application of the method requires the evaluation of lower bounds on two elgenvalues, $\alpha$ and $\beta$, which arise in an associated efgenvalue problem. While these eigenvalue bounds are relatively simple to obtain for scattering by a static central potential, no methods for obtaining bounds on a for scattering by a compound system have been developed.

The determination of a lower bound on $\beta$ gives an upper bound on $(-k \cot \eta)^{-1}$. The determination of this upper bound through the Kato approach has however now been rendered largely academic by the development of a technique 8,9 which does not require the evaluation of matrix elemerts of the square of the Hamiltonian, as does the Kato approach. This technique, incidentally, is a fortiori applicable to (I).

The Kato method as originally formulated was explicitly restricted to one channel processes, and could therefore not be applied to (3), (4), or (5). The present paper concerns itself with the development of extensions of the Kato method which will allow 1 t to be applied to (3); further, explicit methods will be given which will often allow one to obtain lower bounds on $\alpha$ and on $\beta$. [These extensions can also be used, in princlple, for (4), and can probably be extended to (5), but these cases have not as yet been seriously examined.] Now work in progress shows 10 that the techniques referred to can be applied to (3), (4), and (5). One therefore has 
a. very much simpler method for obtaining an upper bound on $(-\mathrm{k} \text { cot } \eta)^{-1}$ for case (3) than can be realized by the present paper. The present work is still justified because one would of course like very much to be able to determine the other bound. (It is of course also preferable not to have to truncate the potential.) Since it entails very little additional Iabor, we will actually obtain both bounds for (3).

\section{MATRIX FORMULATION OF THE VARIATIONAL PRINCIPIE}

We begin by showing how the one dimensional scattering problem with potential $V(x)$ can be written as a differential equation in matrix form. We have

$$
-\frac{\hbar^{2}}{2 n} \frac{d^{2} u(x)}{d x^{2}}+[v(x)-E] u(x)=0
$$

where we take $V(x)$ to be real and to vanish more rapidly than $I / x$ for $|x| \rightarrow \infty$. It is evident that there are two channels, corresponding to $x>0$ and to $x<0$, respectively, which are coupled by the potential, and consequently three independent parameters are required to specify the asymptotic effects of the scattering process.

It is convenient to recast Eq. (I) in matrix form so that the three unknown parameters appear as the three independent elements of a single matrix quantity. This can be accomplished by expressing $u(x)$ and $V(x)$ as sums of even and odd functions,

$$
u(x)=u_{e}(x)+u_{0}(x), \quad v(x)=v_{e}(x)+v_{0}(x)
$$


where

$$
u_{e, o}(x) \equiv \frac{1}{2}[u(x) \pm u(-x)]
$$

and

$$
v_{e, o}(x) \equiv \frac{1}{2}[V(x) \pm V(-x)]
$$

$V_{e}$ and $V_{0}$ wlll clearly also vanish more rapldly than $l / x$ as $|x| \rightarrow \infty$. If we replace $x$ by $-x$ in $E q .(1)$, add and subtract the derived equation and Eq. (1), and use Eqs. (3) and (4), we obtaln the single matrix equation ${ }^{11}$

$$
(\underset{m}{H}-\mathrm{E}) \underline{u}=-\frac{\hbar^{2}}{2 m} \frac{d^{2} \underline{u}}{d x^{2}}+[\underset{m}{V}-E] \underset{m}{u}=0
$$

where
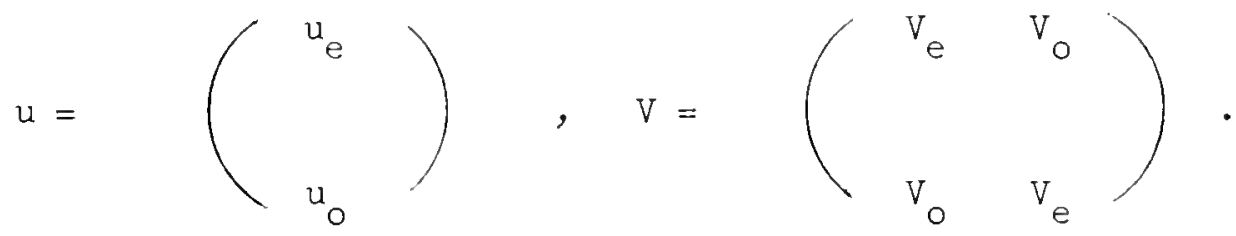

Note that the channels, which are still coupled in general, now correspond to the even and odd portions of the wave function rather than to $x>0$ and $x<0$. We can restrict the range of the independent variable to $x \geqq 0$ since the components of $u$ are even and odd functions of the coordinate. The boundary condtions at the orlgin are, obviously,

$$
u_{0}=0, d u_{e} / d x=0
$$


We see then that one can conveniently formulate one dimensional scattering in terms of a schroedinger equation with $\mathrm{V}(\mathrm{x})$ a real hermitian $2 \times 2$ matrix. For the formal development which follows, we will not restrict ourselves to matrix potentials of the above form but rather we will take $\underset{m}{V}(\mathrm{x})$ to be an arbitrary real hermitian $2 \times 2$ matrix whose elements fall off faster than $I / x$. The Indices will therefore be $I$ and 2 rather than $e$ and 0 . The results to be obtained are actually valid for $\mathrm{V}$ an $N \times \mathbb{N}$ matrix, that is, for an $N$ channel process; we set $N=2$ only as a matter of convenience since it slightly simplifies the discussion and the notation. Many elastic scattering processes involving compound systems can be written in a slightly generalized form of Eq. (5).

We will now derive a variational principle for scattering which can be described by Eq. (5). The asymptotic form of $\mathbf{u}(x)$ can be written as

$$
\begin{aligned}
& \underset{m}{u}(x) \rightarrow \underline{a}_{-\theta} e^{-i(k x+\theta)}+\underline{b}_{\theta} e^{+i(k x+\theta)}, \\
& k^{2} \equiv 2 m / \hbar^{2} .
\end{aligned}
$$

The utility of the parameter $\theta$, where $0 \leqq \theta<\pi$ but is otherwise arbitrary, will become apparent later. It is convenient to rewrite Eq. (8a) in a form that involves standing waves rather than traveling waves, since we wlll then be able to deal with hermitian rather than unitary matrices,

$$
\begin{aligned}
& u(x) \rightarrow e_{m \theta} \cos (k x+\theta)-i j_{\theta} \sin (k x+\theta),
\end{aligned}
$$

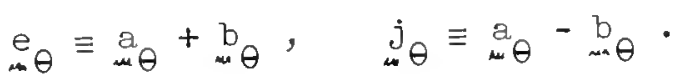


The amplitude vector for the outgoing waves, $b_{\theta}$ is related to that for the incoming waves, a $\theta$, by the scattering matrix $\mathrm{S}_{\Theta}$, through

$$
\underset{m \theta}{b_{\theta}}=-S_{\theta} \underset{m \theta}{a}
$$

The vectors $e_{m} \theta$ and $\dot{j}_{\theta}$ are related by the susceptance matrix $\underset{\sim \theta}{B}$ (so called because it is analogous to the susceptance matrix in network theory),

$$
j_{\theta}=-i_{m \theta} e_{m}^{e}
$$

From $\mathrm{Eq} .(9)$ and $(10)$ It can be seen that $\mathrm{B}_{\theta}$ is the inverse of the Cayley transform of $\underset{m}{S}$,

$$
\underset{m \theta}{B}=i\left(1+S_{m}\right)\left(I-S_{m}\right)^{-1}
$$

It can be shown that $\underset{m \theta}{S}$ is unitary and symmetri $\mathrm{c}^{12}$ and as a consequence that $\underset{m \theta}{\mathrm{B}}$ is hermitian and symmetric and therefore real. Equation (1I) can now be used to eliminate $j_{m}^{j}$ in Eq. (9a), giving rise to

$$
\underset{m}{u}(x) \rightarrow e_{m}^{e} \cos (k x+\theta)-B_{m \theta} \underset{m \theta}{e} \sin (k x+\theta) .
$$

We now introduce a trial wave function $u_{n t}(x)$ which is required to have the asymptotic form

$$
u_{m} t(x) \rightarrow e_{m} \cos (k x+\theta)-B_{m} \theta t e_{m} \sin (k x+\theta)
$$

as $x \rightarrow+\infty$, that $i s$, the amplitude vector $e_{\theta}$ is the same as that of the exact function, but the unknown $\underset{m \theta}{B}$ is replaced by $\underset{m e t}{B}$. In addition, we impose the boundary condition

$$
\underset{m}{u} u_{m t}^{d} u_{t} / d x=\left(d_{m}^{t} / d x\right) u_{m}
$$


at $x=0$, and we require that $u_{m}$ and $d_{u t} / d x$ be continuous in the range $0<x<\infty$. It is to be noted that all of the boundary conditions imposed on $u_{t}$ are also satisfied by the exact function. In order to arrive at a variational prineiple, consider the relation (all integrals extend from 0 to $\infty$, and the limits will henceforth be omitted)

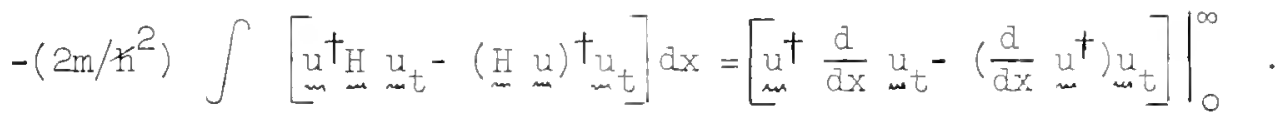

Using Eqs. (13) and (5), the boundary condition at the origin, and the symmetry of $\mathrm{B}_{\boldsymbol{\theta}}$, we obtain from $\mathrm{Eq}$. (15)

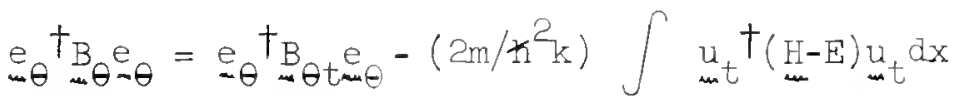

$$
\begin{aligned}
& \left.+\left(2 m / x^{2} x\right) \int \underset{m}{w}+\underset{m}{H-E}\right)_{m} d x, \\
& w_{m} \equiv u_{n t}-u_{m}
\end{aligned}
$$

The first two terms on the right hand side of $\mathrm{Eq}$. (I6a) can be evaluated directly from the trial function, and the third term is of second order in the difference between the trial function and the exact function. The first two terms therefore constitute a vaciational approximation for

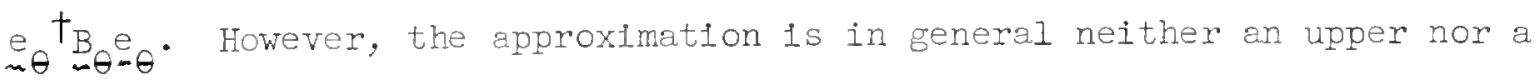
lower bound on the exact value. In the next section we will derive rigorous second-order upper and lower bounds on the error term, the third term on the right hand side of $\mathrm{Eq} \cdot(16 \mathrm{a})$. 


\section{RIGOROUS BOUNDS ON THE ERROR TERM}

In this section we wll derive rigorous upper and lower bounds on the error term in Eq. (16a), following a procedure analogous to that derived by Kato. ${ }^{1}$ we begin by considering the associated eigenvalue problem,

$$
\left({ }_{m}-E\right) \phi(x)-\mu_{m}(x) \phi(x)=0
$$

In Eq. (17), $\rho(x)$ is required to be a real, positive definite hermitian matrix each of whose elements mast fall off faster than $1 / x$ as $|x| \rightarrow \infty$. In particular, we are interested in discrete eigenvalues $\mu_{n}$, of which there will be an infinite number, defined by the condition that $\mathrm{H}$ is a hermitian operator when sperating on the corresponding eigenfunctions, $\phi_{n}$, or on $w$ [efined by Eq. (16b)], that is,

$$
\begin{aligned}
& \int\left[\phi_{m}^{+}{ }_{m}^{H} \phi_{n}-\left(\underline{H} \phi_{m}\right)^{+} \phi_{n}\right] d x=0 \\
& \int\left[w^{\dagger} H \phi_{n}-\left(w_{w}^{H}\right)^{\dagger} \phi_{n}\right] d x=0
\end{aligned}
$$

It follows from the hermitian character of $\mathrm{H}$ that the associated eigenfunctions are orthogonal with respect to the weight factor $\rho$ and that the associated eigenvalues are real,

$$
\begin{aligned}
& \int \phi_{m}^{+} \rho \phi_{n} d x=\left(\hbar^{2} \mathrm{k} / 2 m\right) \delta_{m n}, \\
& \mu_{n}^{*}=\mu_{n} .
\end{aligned}
$$


The normalization in Eq. (19a) has been chosen for later convenience. In addition we require that the $\phi_{n}$ satisfy the boundary conditions at $x=0$,

$$
\begin{aligned}
& \phi_{m} \dagger d \phi_{n} / d x=\left(d \phi_{m} \dagger / d x\right) \phi_{n}, \\
& \phi_{n}^{\dagger} \underset{m}{d w} / d x=\left(d \phi_{n} \dagger / d x\right)_{m}^{w} .
\end{aligned}
$$

In analogy with $\mathrm{Eq} \cdot(13), \oint_{\mathrm{n}}$ has the asymptotic form as $x \rightarrow \infty$,

$$
\phi_{m n} \rightarrow \underset{m n}{e} \cos k x-B\left(\mu_{n}\right) \underset{m n}{e} \sin k x
$$

From Eqs. (13), (14) and (16b) it follows that the asymptotic form of $\underset{\mathrm{m}}{\mathrm{w}}$ is

$$
\underset{m}{w} \rightarrow\left(B_{m}-\underset{n \theta t}{B}\right) \underset{m \theta}{e} \sin (k x+\theta) .
$$

Using Green's theorem and Eqs. (20), (21), and (22) to evaluate the integrals in Eqs. (18) we find that Eq. (18) can be satisfied only if $B\left(\mu_{n}\right)$ satisfies

$$
\mathrm{B}_{m}\left(\mu_{n}\right) e_{-n}=-\cot \theta \underset{m n}{e}
$$

Thus the associated eigenfunctions are also eigenmodes, i.e., their amplitude vectors $e_{w}$ are eigenvectors of the susceptance matrix $\underset{m}{B}\left(\mu_{n}\right)$. The $\mu_{n}$ are just the values of $\mu$ for which one or another of the eigenvalues of the susceptance matrix is equal to $-\cot \theta$.

We will. now proceed to derive a monotonicity theorem required in subsequent developments. In principle, we can find solutions $\phi(x)$ of 
Eq. (17) for arbitraxy $\mu$ if we no longer impose the boundary conditions (18). In particular, we can find 2 eigenmodes $\phi_{i}(x)(i=1,2)$ for an arbitrary value of $\mu$ which are distinguishea by their asymptotic forms,

$$
\phi_{i}(\mu) \rightarrow e_{i}(\mu)\left[\cos k x-\zeta_{i}(\mu) \sin k x\right] .
$$

In Eq. (24) $\zeta_{i}(\mu)$ is the $i^{\text {th }}$ eigenvalue of $\underset{m}{\mathrm{E}}(\mu)$. It shoula be noted that the eigenmode solutions of Eq. (17) are employed here only in the formal development. Presumably, it is more tifficult to find such solutions than to solve the original problem, Iq. (5). At this point it is convenient to relabel the associated eigenfunctions with two indices. The associated eigenvalues $\mu_{n i}$ are then definea by the relation

$$
\zeta_{i}\left(\mu_{n i}\right)=-\cot (\theta+n \pi)
$$

where the index $i$ labels the eigenmodes and the corresponding associated eigenfunctions have the asymptotic form

$$
\oint_{n i} \rightarrow e_{m i}\left(\mu_{n i}\right)[\cos k x+\cot (\theta+n \pi) \sin k x] .
$$

Consider the eigenmode solutions of $\mathrm{Eq}$. (17) for two different values of $\mu$, to be denoted by $\mu^{\prime}$ and $\mu^{\prime \prime}$, without applying the boundary conditions (18), and form the expression

$$
\begin{aligned}
& -\left(2 m / \hbar^{2}\right) \int\left[\phi_{i}^{\dagger}\left(\mu^{\prime}\right) \underset{m}{H} \phi_{m}\left(\mu^{\prime \prime}\right)-\left(\omega_{\omega}^{H} \phi_{i}\left(\mu^{\prime}\right)\right)^{\dagger} \phi_{j j}\left(\mu^{\prime \prime}\right)\right] d x \\
& =\left(2 m / \hbar^{2}\right)\left(\mu^{\prime *}-\mu^{\prime \prime}\right) \int \phi_{i}^{+}\left(\mu^{\prime}\right) \rho \phi_{m j}\left(\mu^{\prime \prime}\right) d x \\
& =\left.\left[\phi_{i}^{\dagger}\left(\mu^{\prime}\right) \frac{\mathrm{d}}{\mathrm{dx}} \phi_{j}\left(\mu^{\prime \prime}\right)-\frac{\mathrm{d}}{\mathrm{dx}} \phi_{i}^{+}\left(\mu^{\prime}\right) \phi_{j}\left(\mu^{\prime \prime}\right)\right]\right|_{0} ^{\infty}
\end{aligned}
$$


where we have made use of Eqs. (5) and (17). Using Eqs. (20) and the asymptotic form of $\phi_{i}(\mu)$ given by $\mathrm{Eq} \cdot(24)$, we may rewrite $\mathrm{Eq} \cdot(27)$ as

$$
\begin{aligned}
& \left(2 m / \hbar^{2}\right)\left(\mu^{\prime *}-\mu^{\prime \prime}\right) \int \phi_{i}^{\dagger}\left(\mu^{\prime}\right) \rho \phi_{m}\left(\mu^{\prime \prime}\right) d x \\
& =k e_{i} e^{\dagger}\left(\mu^{\prime}\right) e_{m j}^{e}\left(\mu^{\prime \prime}\right)\left[\zeta_{i}\left(\mu^{\prime}\right)-\zeta_{j}\left(\mu^{\prime \prime}\right)\right] .
\end{aligned}
$$

Consider $\mathrm{Eq}$. (28) with $\mu^{\prime}$ and $\mu^{\prime \prime}$ real but otherwise arbitrary and with $j=i$. Let the eigenphase shift $\delta_{i}(\mu)$ be defined by

$$
\zeta_{i}(\mu)=-\cot \delta_{i}(\mu)
$$

Substituting Eq. (29) in Eq. (28) and taking the limit $\mu^{\prime \prime} \rightarrow \mu^{\prime}$, we obtain

$$
\left.a \delta_{i} / d \mu=\left(2 m / \varkappa^{2}\right) \sin ^{2} \delta_{i} \int \phi_{i}^{+} \rho \phi_{m i} d x / \underset{-i}{\left(k e_{i}\right.} t_{e_{i}}\right) \geqq 0 .
$$

The inequality in Eq. (30) follows from the positive definite character of $\rho_{m}$. Thus the eigenphase shifts are monotonic increasing functions of $\mu($ for $k \neq 0)$.

We now proceed to derive rigorous bounds on the error term in Eq. (16a). Suppose that ${\underset{m}{m}}_{\text {and }} \mathrm{g}_{\mathrm{m}}$ are functions which either have the same phase shift as the $\phi_{\text {mi }}$ or fall off asymptotically faster than $1 / x$. We define expansion coefficients $a_{n i}$ and $b_{n i}$ by tine relations

$$
a_{n i}=\left(2 m / x^{2} x\right) \int \phi_{n i}+\rho f_{m} d x,
$$




$$
b_{n i} \equiv\left(2 m / K^{2} k\right) \int \phi_{n i}^{+} p_{m}^{g} d x
$$

It can then be shown that the associated eigenfunctions form a complete set $^{13}$ in the sense that

$$
\begin{aligned}
& \int f_{m}^{t} \rho f d x=\left(x^{2} k / 2 m\right) \sum_{n, i} a_{n i}^{*} a_{n i} \\
& \int f_{m}^{\dagger} \rho \underset{m}{g} d x=\left(\hbar^{2} k / 2 m\right) \quad \sum_{n, i} a_{n i}^{*} b_{n i} \\
& \int g_{m}^{\dagger} \rho g d x=\left(\hbar^{2} k / 2 m\right) \sum_{n, i} b_{n i}^{*} b_{n i}
\end{aligned}
$$

If we now set $\underset{m}{f}=\underset{m}{W}$ and $\underset{m}{g}=-\rho_{m}^{-1}(\underset{\mu}{H}-E)_{m}$, it follows from Eqs. (3I) that $a_{n i}$ and $b_{n i}$ are related by

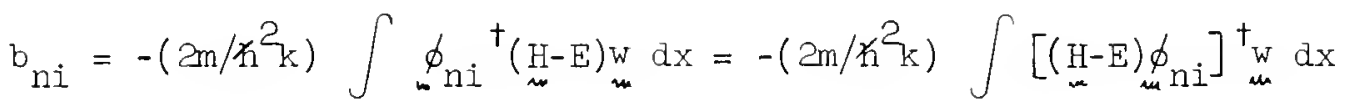

$$
\begin{aligned}
& =-\mu_{n i}\left(2 m / x^{2} k\right) \int \phi_{n i}^{\dagger} \rho w d x=-\mu_{n i} a_{n i},
\end{aligned}
$$

provided $\rho_{\mathrm{m}}$ is chosen such that $\underset{\mathrm{m}}{\mathrm{g}}$ falls off asymptotically faster than $l / x$. Eqs. (32) can now be written in the form 


$$
\begin{aligned}
& \int w_{w}^{\dagger} \rho_{m} w_{m} d x=\left(\hbar^{2} k / 2 m\right) \quad \sum_{n, i} \mu_{n i}^{-2} b_{n i}^{*} b_{n i}, \\
& \int w_{m}^{\dagger}(\underset{m}{H-E})_{m} d x=\left(\hbar^{2} k / 2 m\right) \sum_{n, i} \mu_{n i}^{-1} b_{n i}^{*} b_{n i},
\end{aligned}
$$

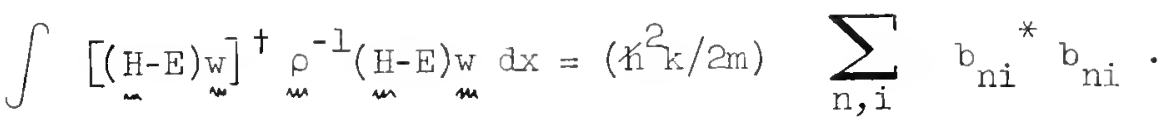

Let $\alpha_{\theta}$ be the smallest positive eigenvalue and $-\beta_{\theta}$ be the smallest (in absolute value) negative eigenvalue. It follows from Eqs. (34) that

$$
\begin{aligned}
& -\beta_{\theta}^{-1} \epsilon_{\theta}^{2} \leqq \int w_{m}^{\dagger} \underset{m}{(H-E) w} d x \leqq \alpha_{\theta}^{-1} \epsilon_{\theta}{ }^{2} \text {, }
\end{aligned}
$$

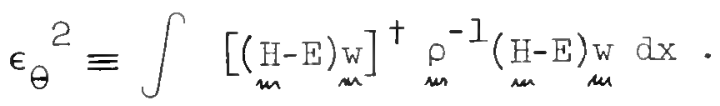

We have thus obtained rigorous upper and lower bounds on the error term in Eq. (16a). The quantity $\epsilon_{\theta}^{2}$ is of second order in the difference between the trial function and the exact function, and can be calculated from the trial function since it follows from Eqs. (5) and (16b) that

$$
\underset{\mu}{(H-E})_{\mu}^{W}=(\underset{\mu}{H}-E) u_{\mu}
$$


The problem otill remains of course of obtaining lower bounds on $\alpha_{\theta}$ and on $\beta_{\theta}$. It is to be recalled that it is possible to obtain close bounds on $\int_{m}^{W} \underset{m}{\dagger}(\underset{m}{H-E}) \underset{w}{w}$ even with quite crude lower bounds on $\alpha_{\theta}$ and $\beta_{\theta}$, since $\epsilon_{\theta}^{2}$ is a second order quantity.

$$
\text { 4. BOUNDS ON } a_{\theta} \text { AND ON } \beta_{\theta}
$$

In this section we will indicate a rather general method for obtaining lower bounds on $\alpha_{\theta}$ and on $\beta_{\theta}$ for cases covered by Eq. (5). The monotonicity theorem, Eq. (30), states that the eigenphase shifts $\delta_{i}(\mu)$ are mollotonic increasing functions of $\mu$. The labeling of the eigenphase shifts is essentially arbitrary, but the application of this theorem requires that we keep track of the labeling as $\mu$ is varied. Since $\mu$ is a. continuous variable, this presents no difficulty except at crossing points where $\delta_{i}(\mu)=\delta_{j}(\mu), i \neq j$. However, we are interested in the weaker statement

$$
\mu_{n-1, i} \leqq \mu_{n i} \leqq \mu_{n+1, i}
$$

where $\mu_{n i}$ is the value of $\mu$ for which $\delta_{i}\left(\mu_{n i}\right)=\theta+n_{\pi}$. Eq. (37) holds in spite of the ambiguity in labeling at crossing points, since no matter which curve is continued into which, both continue to rise monotonically. The relationship between $\alpha_{\theta}$ and $\beta_{\theta}$ and the eigenphase shifts is iliustrated for a typical case in Figure l. If we define $\alpha_{\theta i}$ and $-\beta_{\theta i}$ to be the smallest positive eigenvalue and smallest (in absolute value) negative eigenvalue, respectively, associated with the $i^{\text {th }}$ eigenmode, then $\alpha_{\theta}$ is the smaller of the $\alpha_{\theta i}$ and $\beta_{\theta}$ the smaller of the $\beta_{\theta i}$. Thus there is only one $\alpha_{\theta}$ and one $\beta_{\theta}$. 


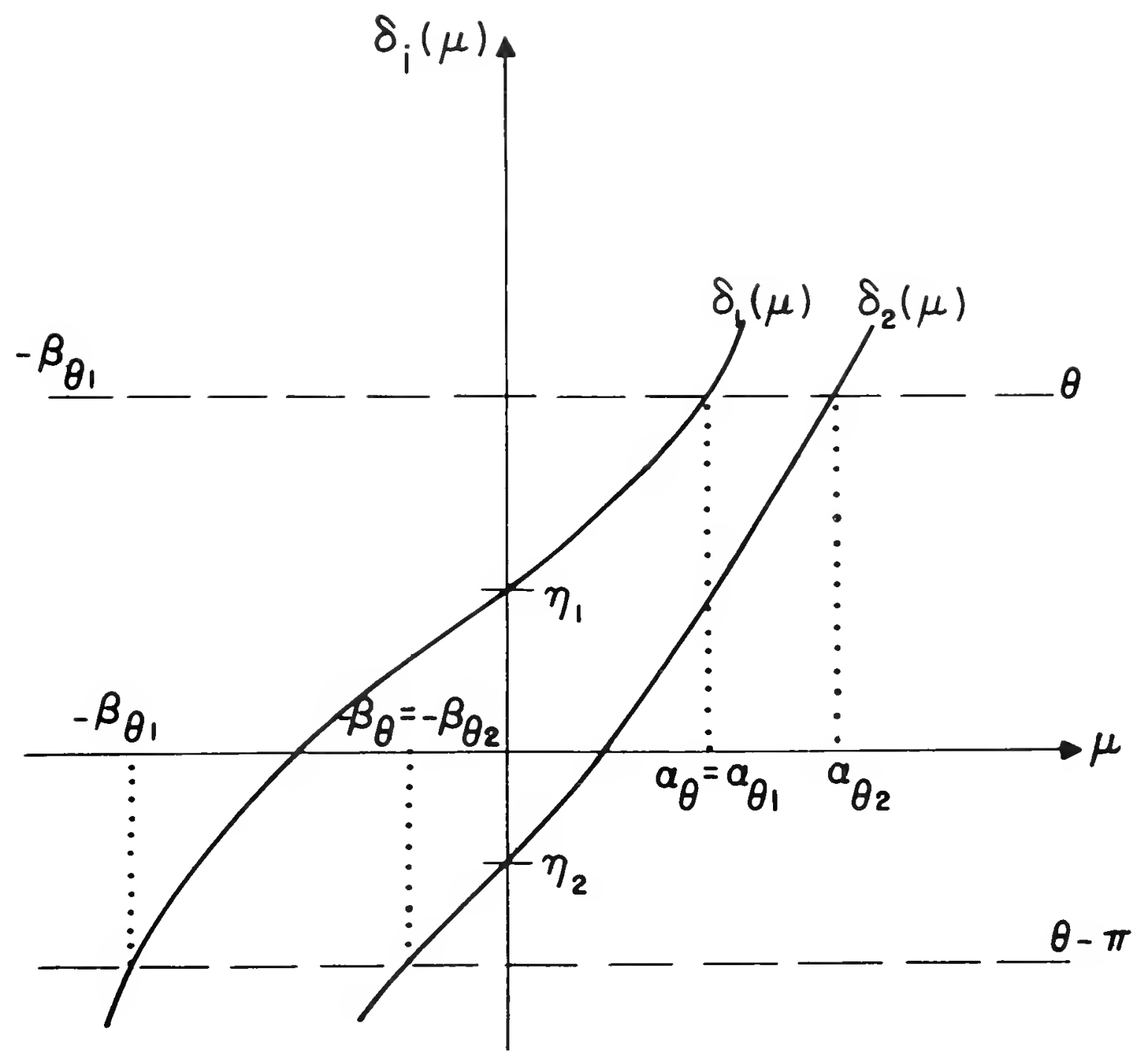

Figure 1

Relationship between $\alpha_{\theta}$ and $\beta_{\theta}$ and the eigenphase shifts of the associated eigenvalue problem, $\delta_{i}(\mu)$. The eigenphase shifts of the original problem are $\eta_{1}$ and $\eta_{2}$. 
Figure I demonstrates the relationship between $\alpha_{\theta}$ and $\beta_{\theta}$ and the eigenphase shifts, but these eigenphase shifts are presumably not known. We must therefore devise a method for obtaining lower bounds on $\alpha_{\theta}$ and $\beta_{\theta}$. To this end, we introduce a comparison potential $\underset{w}{\mathrm{~V}}$ such that $\underset{m}{\Delta} \underset{m}{V}=\underset{m c}{V}-\underset{m}{V}$ is non-positive and for which the associated eigenvalue problem can be solved exactly, or for which bounds can be obtained. We will now show that the eigenphase shifts corresponding to the comparison potential are upper bounds on the eigenphase shifts corresponding to the true potential for any given value of $\mu$. Under the substitutions

$$
\begin{aligned}
& \underset{m}{\mathrm{H}} \rightarrow \underset{m}{\mathrm{H}}-\mu \underset{m}{\mathrm{O}} \\
& \underset{m}{\mathrm{H}}-\mu_{m} \underset{m}{\mathrm{P}} \rightarrow \underset{m}{\mathrm{H}}-\mu \underset{m}{\rho}+\lambda \underset{m m}{\Delta} \underset{m}{\mathrm{~V}},
\end{aligned}
$$

the derivation which led to Eq. (30) gives

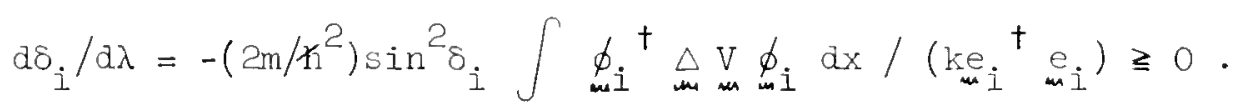

The inequality follows from the non-positive character of $\Delta \underset{w}{\mathrm{~V}}$. The eigenphase shift $\delta_{u i}(\mu)$ corresponding to the comparison potential is obtained when $\lambda=1$, and $\delta_{i}(\mu)$ when $\lambda=0$. It follows from $\mathrm{Eq}$. (39) that $\delta_{u i}(\mu)$ is an upper bound on $\delta_{i}(\mu)$,

$$
\delta_{u i}(\mu) \geq \delta_{i}(\mu) .
$$

As before, we are required to keep track of the eigenphases as $\lambda$ is varied continuously from zero to one. The only difficulty occurs at a possible crossing point, but $\delta_{i}(\mu, \lambda)=\delta_{j}(\mu, \lambda)$ already implies that these eigen- 
phase shifts are greater than the true values and they can only increase as $\lambda$ approaches one. Eq. (40) is therefore valid in any case.

By introducing another comparison potential such that $\Delta \mathrm{m}_{\mathrm{m}}$ is nonnegative, it can be shown in a similar fashion that the corresponding eigenphase shifts $\delta_{\ell_{i}}(\mu)$ are lower bounds on the $\delta_{i}(\mu)$,

$$
\delta_{\ell i}(\mu) \leqq \delta_{i}(\mu) .
$$

The way in which the bounding curves obtained from the comparison potentials can be used to calculate lower bounds $\alpha_{\theta l}$ and $\beta_{\theta l}$ on $\alpha_{\theta}$ and on $\beta_{\theta}$, respectively, is illustrated for a typical case in Figure 2 . It is clear from Figure 2 that if the comparison potentials do not approximate the actual potential fairly closely, it may not be possible to obtain lower bounds on $\alpha_{\theta}$ and on $\beta_{\theta}$. For example, if the curve labeled $\delta_{u I}(\mu)$ in Figure 2 were to cross the dashed line $\delta_{i}(\mu)=\theta$ at a point where $\mu$ is negative, then it would $n+$, be possible to obtain a lower bound on $\alpha_{\Theta}$.

In general it is possible, $a_{i} \equiv$ chall show, to calculate lower bounds on $\alpha_{\theta}$ and on $\beta_{\theta}$ by using com rison potentials and a weight function $\rho$ which are diagonal. In that $\left(\right.$ se, the bounding curves $\delta_{u i}(\mu)$ and $\delta_{\ell i}(\mu)$ are obtained by solving 4 singl -channel problems rather than 2 2-channel problems. The consequences of this result are far reaching, in that very few truly multichannel problems can be solved at all, even with simple comparison potentials. On the other hand, the possibility of using diagonal comparison potentials implies that it is no more difficult to obtain bounds in the multichannel problem than in the singlechannel problem, though it may be much more tedious, and thus the power 


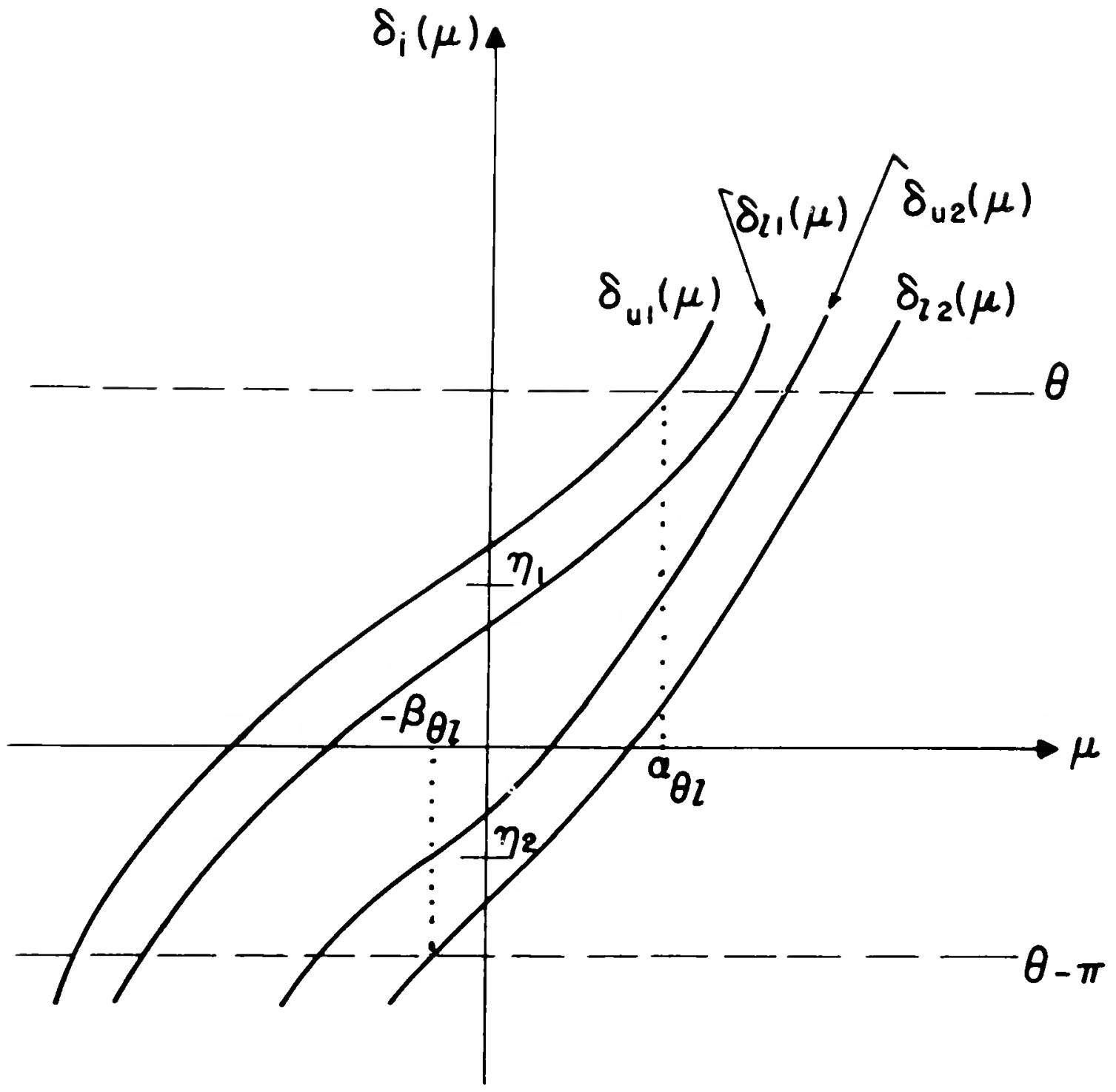

Figure 2

Use of comparison potentials to obtain lower bounds, $\alpha_{\theta \ell}$ and $\beta_{\theta \ell}$, on $\alpha_{\theta}$ and $\beta_{\theta}$, respectively. $\delta_{u i}(\mu)$ and $\delta_{\ell i}(\mu)$ are derived from the comparison potentials and are upper and lower bounds, respectively, on $\delta_{i}(\mu)$. 
of the method is greatly enhanced. Furthermore, the Kato method for obtaining bounds in the single-channel case can be used to obtain bounds on the eigenphase shifts of the comparison potentials, or the singlechannel comparison potential problems could even be solved numerically. We will now consider the requirements that must be imposed on the elements of $V_{c}(x)$ if it is taken to be aiagonal. Writing

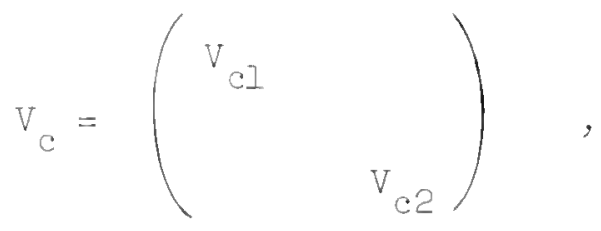

we have

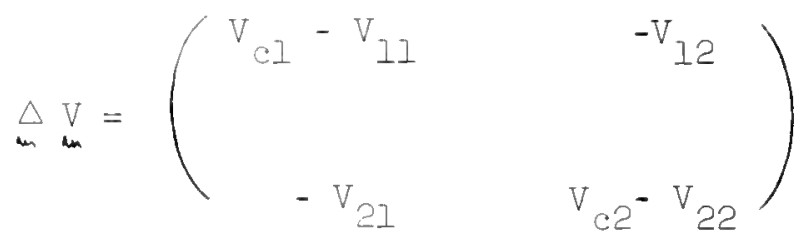

It is then trivial to show that if $\triangle \mathrm{V}$ is to be non-negative, we must have

$$
V_{c 1} \geqq V_{11}+\left|v_{12}\right|, \quad V_{c 2} \geqq V_{22}+\left|v_{12}\right|
$$

while if $\triangle \Delta_{m}$ is to be non-positive, we must have

$$
V_{c 1} \leqq V_{11}-\left|V_{12}\right| \quad, \quad V_{c 2} \leqq V_{22}-\left|V_{12}\right|
$$

The above conditions are necessary but not sufficient for obtaining lower bounds on $\alpha_{\theta}$ and on $\beta_{\theta}$, since we have already seen that it is possible 
that no diagonal comparison potential may be sufficiently close to $\mathrm{V}$ to be able to determine bounds.

\section{METHOD OF OBTAINING BOUNDS}

We are now in a position to apply the foregoing results to the problem of obtaining rigorous upper and lower bounds on the independent elements of the scattering matrix. From Eqs.(16) and (35), we can obtain expressions which are upper and lower bounds on a quadratic form of the susceptance matrix,

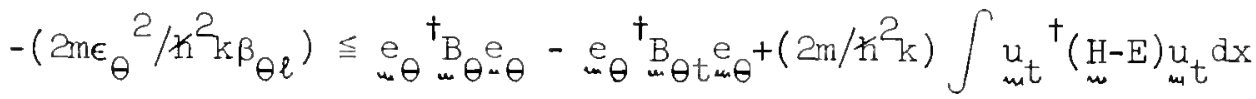

$$
\begin{aligned}
& \leqq\left(2 m \epsilon_{\theta}^{2} / \hbar^{2} k a_{\theta \ell}\right), \\
& \epsilon_{\theta}^{2} \equiv \int\left[(\underset{\omega}{H}-E) u_{t}\right]^{\dagger} p_{m}^{-I}(H-E) u_{m} d x
\end{aligned}
$$

where $\alpha_{\theta \ell}$ and $\beta_{\theta \ell}$ are lower bounds on $\alpha_{\theta}$ and on $\beta_{\theta}$, respectively. We thus have variational bounds on $e_{\theta}{ }^{\dagger} B_{\theta} e_{\theta}$. The bound property enables us to improve the approximations by incorporating variational parameters into $u_{w t}$. As opposed to the situation in the usual variational calculation, we do not have to find the stationary values of the parameters (it is preferable but not essential) but can rather evaluate the bound for a few values of the parameter and unambiguously choose the best result. This makes feasible the use of variational parameters which are 
not linear. $\theta$ can be thought of a such a parameter, though in the present paper we will not take full advantage of this property.

As an alternative procedure, we could adjust the variational parameters to minimize the quantity $\epsilon_{\theta}{ }^{2}$ given by $\mathrm{Eq}$. (47). While this procedure is simpler, in general it yields inferior bounds and consequently it will not be followed in this paper. With reference to Figure 2, $\theta$ should be chosen to provide the greatest lower bounds on $\alpha_{\theta}$ and on $\beta_{\theta}$. In the single-channel problem $\theta$ can be chosen differently for $\alpha_{\theta}$ and for $\beta_{\theta}$, since the upper and lower bounds on the phase shift are obtained by solving two distinct problems. However, in the multi-channel case the susceptance matrices $\underset{m}{B_{\theta}}$ corresponding to two different values of $\theta$ are related in such a complex manner that it does not appear feasible to obtain upper and lower bounds on the 3 independent parameters of the scattering matrix by using different values of $\theta$ for the upper and lower bounds on the quadratic forms $\operatorname{ee}_{-\theta}^{+} B_{\mu} e_{m}$.

In order to obtain bounds on the 3 distinct elements of ${ }_{\mu}{ }_{\theta}$, we must evaluate the right hand sides of Eqs. (46) with 3 different choices of the trial function which differ in the amplitude vector $e_{\theta}$. Note that the different values of $e_{\theta}$ are not required to be linearly independent

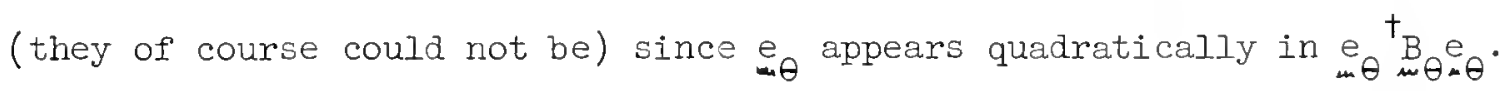
Rather, the resulting inequalities in the 3 independent elements of $B_{\theta}$ must be linearly independent. Since there are two bounds for each choice of $e^{\prime} \theta^{\prime}$ there are 6 problems in all.

Since the scattering matrix $\underset{\mu}{\mathrm{S}}$ (the scattering matrix $\underset{\mathrm{m}}{\mathrm{S}}$ as normally defined is $\operatorname{S}_{\boldsymbol{m}}$ with $\theta=0$ ) can be calculated from ${ }_{\boldsymbol{m}}^{\mathrm{B}}$, the 3 independent elements of $\underset{\mu \theta}{B}$ are sufficient to determine $\underset{m}{S}$. $\underset{m}{S}$ can also be calculated 
from an alternative set of 3 parameters, the 2 eigenphase shifts and the mixing parameter. This alternative mode of description is useful in many applications.

We now examine the procedure for obtaining bounds on the eigenphase shifts and mixing parameters. Since $\mathrm{B}_{\ominus}$ is hermitian and real, it can be diagonalized by an orthogonal matrix, $\underset{w}{\mathrm{w}}$. The same matrix $\mathrm{T}$ also diagonalizes $\mathrm{S}$, since $\underset{m \theta}{\mathrm{B}}$ is related to $\underset{\mathrm{m}}{\mathrm{S}}$ by a rational fraction. Because $\underset{m}{\mathrm{~S}}$ is unitary, its diagonal form can be written as $\exp (2 i \eta)$ where $\eta_{m}$ is a diagonal matrix whose elements are the eigenphase shifts. Note that when the eigenphase shifts are defined in this way, they do not necessarily vanish in the absence of a potential but rather assume values consistent with the boundary conditions at the origin. The mixing parameter is just the angle of rotation on which $\mathrm{T}$ depends. It follows from Eqs. (8a), (10) and (12) that $\underset{\sim}{\mathrm{B}} \boldsymbol{\theta}$ is related to $\underset{\mathrm{m}}{\mathrm{S}}$ by

$$
B_{m}=i\left(I_{m}+\operatorname{Se}_{m}^{-2 i \theta}\right)\left(I_{m}-S_{m} e^{-2 i \theta}\right)^{-1}
$$

From the foregoing discussion, $\underset{m}{\mathrm{~S}}$ can be represented by

$$
S=\underset{m}{T} \exp (2 i \eta) \underset{m}{T}
$$

Therefore, from $\mathrm{Eq} \cdot(48)$ and (49) $\underset{\mathrm{m}}{\mathrm{B}} \mathrm{can}$ be written as

$$
\underset{\omega}{B}=-\underset{\omega}{T} \cot (\underset{m}{\eta}-\theta) \underset{m}{\widetilde{T}}
$$

The orthogonal matrix $\underset{m}{\mathrm{~m}}$ can be written as

$$
\frac{T}{m}=\left(\begin{array}{cc}
\cos \psi & \sin \psi \\
-\sin \psi & \cos \psi
\end{array}\right),
$$


where $\psi$ is the mixing parameter. Solving $E q .(50)$ and (5I) for $\psi$, $\eta_{1}$, and $\eta_{2}$, we obtain

$$
\begin{aligned}
& \psi=\frac{1}{2} \cot ^{-1}\left[\left(B_{\theta 22}-B_{\theta 11}\right) /\left(2 B_{\theta 12}\right)\right] \text {. } \\
& \eta_{I}=\theta+\cot ^{-1}\left[\frac{1}{2}\left(B_{\theta 22}-B_{\theta 1 I}\right) \cos 2 \psi+B_{\theta 12} \sin 2 \psi-\frac{1}{2}\left(B_{\theta 22}+B_{\theta I I}\right)\right] \\
& \eta_{2}=\theta+\cot ^{-1}\left[-\frac{1}{2}\left(B_{\theta 22^{-}} B_{\theta 11}\right) \cos 2 \psi-B_{\theta 12} \sin \psi-\frac{1}{2}\left(B_{\theta 22}+B_{\theta 11}\right)\right] \text {. }
\end{aligned}
$$

It is clear that by choosing appropriate combinations of upper and lower bounds on the elements of $\mathrm{B}_{\mathrm{m}}$ in Eqs. (52), we can derive upper and lower bounds on $\psi, \eta_{1}$, and $\eta_{2}$.

\section{NUMERICAI EXAMPLES}

In order to test the practicality of the method, we have applied it to the problem of one dimensional scattering by an attractive square well which is asymmetrically placed with respect to the origin, that is, $V(x)=0$ for $x<0$ and for $x>a$, and $V(x)=\left(-\pi^{2} / 2 m\right) U$ for $0 \leqq x \leqq a$, where $U$ is a positive constant. (It should be emphasized that our method is not restricted to potentials which vanish identically.) Eqs. (4) and (6) then give 


$$
\begin{aligned}
V(x) & =-\left(\hbar^{2} / 4 m\right) U\left(\begin{array}{ll}
1 & 1 \\
1 & 1
\end{array}\right) \\
& =\underset{m}{0}
\end{aligned}
$$

where the range of $x$ is now 0 to $\infty$. The trial function within the region of the square well was chosen to be

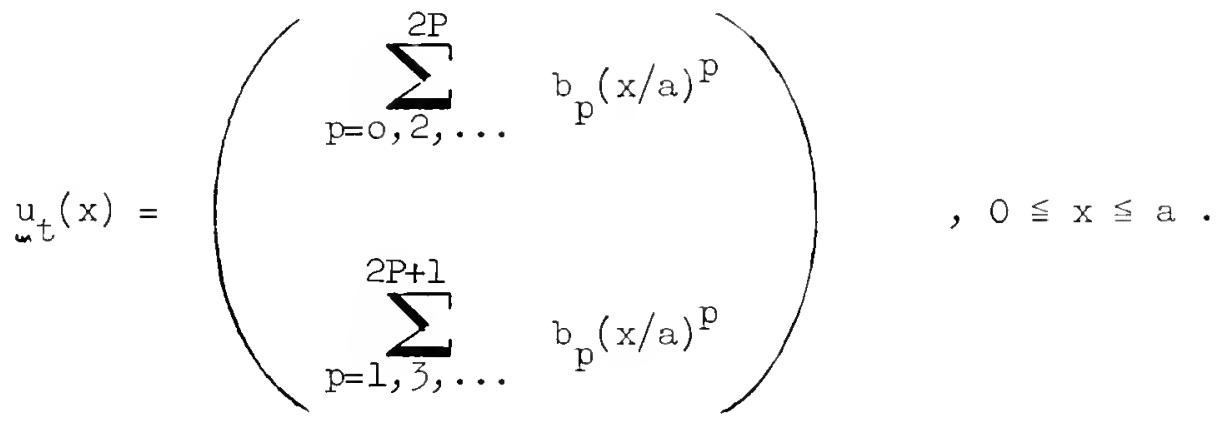

The illogicalness of the unnecessarily restricted form of power series was not recognized until after the numerical calculations had been completed, and it was not then considered worthwhile to repeat the calculations with a less restricted form.

For all $x>a, u_{t}$ was chosen to have the form given by Eq. (14). $u_{\text {ut }}$ must of course be continuous in slope and value at $x=a$.

The comparison potentials which generate non-negative and non-positive matrices $\Delta_{m} \underset{m}{V}$ were taken to be

$$
\begin{aligned}
V_{m C}(x) & =-\left(\hbar^{2} / 2 m\right) U \underset{\omega}{I} & & , \quad 0 \leqq x \leqq a \\
& =0 & & a<x<\infty
\end{aligned}
$$


and

$$
\operatorname{vic}_{\mathrm{c}}(\mathrm{x})=\underset{m}{0}, \quad 0 \leqq x \leqq \infty
$$

respectively. p was chosen as

$$
\rho=\rho_{0} \underset{m}{I}, \quad 0 \leqq x \leqq a, \quad \rho_{m}=\underset{m}{0}, \quad a<x<\infty,
$$

where $\rho_{0}$ is a constant. The eigenphase shifts $\delta_{e}(\mu)$ and $\delta_{0}(\mu)$ corresponding to the lower bound are just the phase shifts associated witr the even and odd solutions, respectively, of the scalar differential equations

$$
\begin{array}{ll}
\frac{a^{2} \phi(x)}{d x^{2}}+k^{2} \phi(x)+\left(2 m / k^{2}\right) \mu \rho_{0} \phi(x)=0, & 0 \leqq x \leqq a \\
\frac{d^{2} \phi(x)}{d x^{2}}+k^{2} \phi(x)=0, & a<x<\infty
\end{array}
$$

while the eigenphase shifts corresponding to the upper bound are obtained from the same equation under the substitution

$$
\mu \rho_{0} \rightarrow \mu_{0}+\left(\hbar^{2} / 2 m\right) U
$$

The phase shifts corresponding to Eq. (57) are given by

$$
\begin{aligned}
& \delta_{e}(\mu)=\pi / 2-k a+\cot ^{-1}[(k a / k a) \cot k a], \\
& \delta_{0}(\mu)=-k a+\tan ^{-1}[(k a / k a) \tan k a] \\
& k \equiv\left(k^{2}+2 m \mu \rho_{0} / \pi^{2}\right)^{\frac{1}{2}}
\end{aligned}
$$


If we plot $\delta^{\prime} e^{(\mu)}$ and $\delta^{\prime}{ }_{0}(\mu)$ versus $2 m \mu p_{0} a^{2} / \hbar^{2}$, we obtain curves each of which lies entirely below the corresponding curve for the exact eigenphase shift. If instead of $\mathrm{Eq} \cdot(60)$ we define $\mathrm{k}$ by

$$
k=\left(k^{2}+2 m \mu \rho_{0} / x^{2}+U\right)^{\frac{1}{2}}
$$

we obtain curves which lie entirely above the corresponding exact curves. These pairs of bounding curves are then used to obtain lower bounds on $\alpha_{\theta}$ and on $\beta_{\theta}$ in accordance with the procedure described in section 4 . The particular value chosen for fo plays no role whatever. ( $\mu$ and $p$ appear only in the combination up. Choosing polarger would tinen give smaller values of $\alpha_{\theta}$ and of $\beta_{\theta}$, but of course the bounds on $e_{\mu}{ }_{\mu}^{\dagger} B_{\theta} e_{\mu}$ would not be affected.)

We will now present some numerical results obtained with the use of the Bendix G-15 digital computer at the General Telephone and Electronics Laboratories, Bayside, Long Island. Bounding curves on $\delta_{e}(\mu)$ and $\delta(\mu)$ calculated from Eqs. (59) and (6I) are plotted in Figures 3 and 4 for $\mathrm{ka}=0.1, \mathrm{Ua}^{2}=1.0$ and in Figures 5 and 6 for $\mathrm{ka}=\pi / 4, \mathrm{Ua}^{2}=\pi^{2} / 4$. The lower bounding curves in Figures 3 and 4 apply also to ka $=0.1$, $\mathrm{Ua}^{2}=0.01$, but in this case the upper bounding curves are so close to the lower bounding curves that the two curves cannot be distinguished from one another on the scale of the drawings. Values of $\theta$ and lower bounds on $\alpha_{\theta}$ and $\beta_{\theta}$ consistent with those curves are listed in Table $I$. In each case, $\theta$ was somewhat arbitrarily chosen to provide approximately equal lower bounds on $\alpha_{\theta}$ and $\beta_{\theta}$. For each choice of ka and of $\mathrm{Ua}^{2}$, three choices were made for $e_{\theta}$, and upper and lower bounds were calculated on the corresponding values of $e_{m}{ }^{\dagger}{ }_{m} e_{m}^{e} e^{e}$. The three choices of $e_{m}$ were 


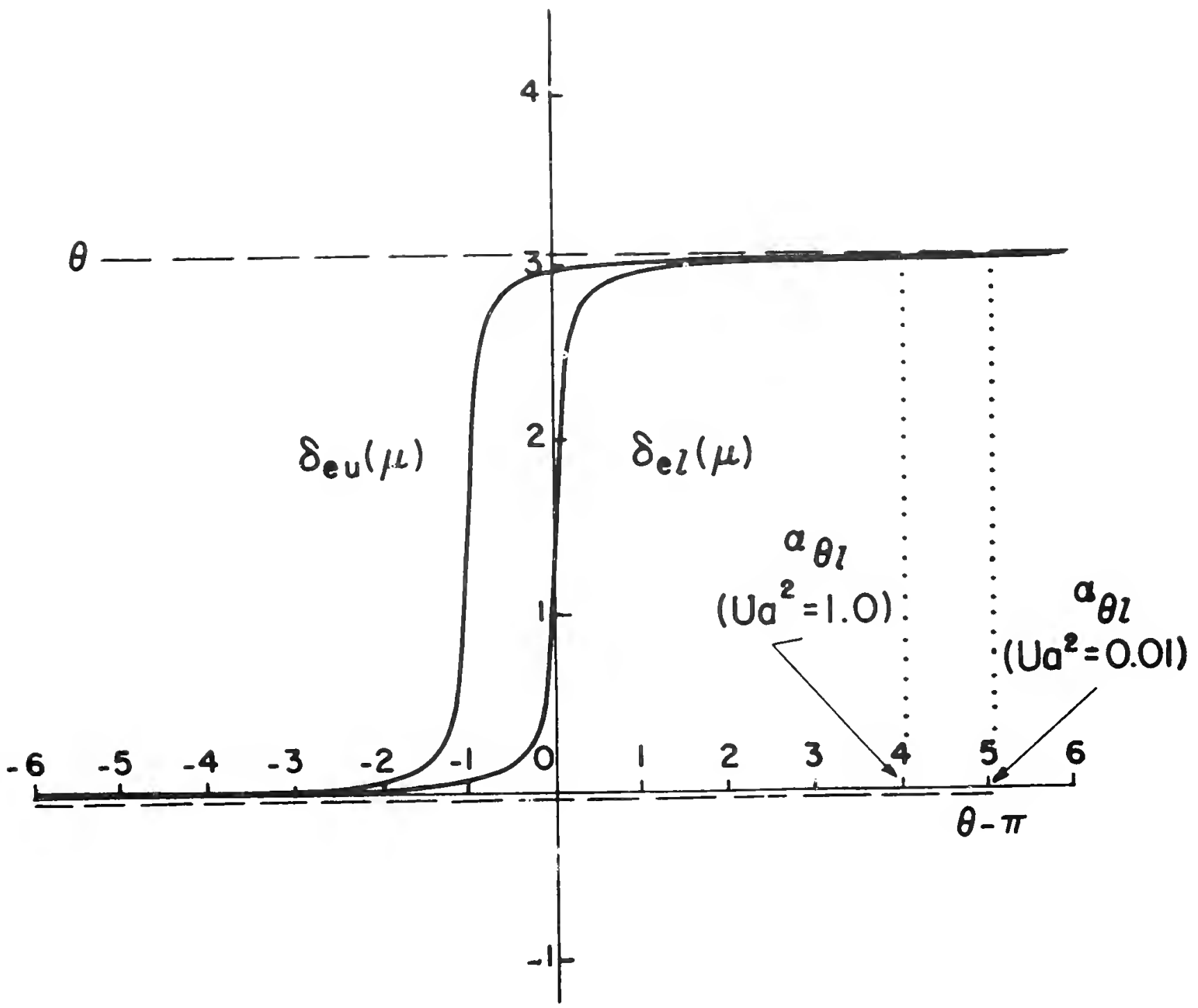

Figure 3

Upper and lower bounds on $\delta_{e}(\mu)$ for $\mathrm{ka}=0.1, \mathrm{Ua}^{2}=1.0$. The lower bound, $\delta_{e \ell}(\mu)$, also applies to $\mathrm{ka}=0.1, \mathrm{Ua}^{2}=0.01$.

The corresponding upper bound, $\delta_{e u}(\mu)$, cannot be distinguished from $\delta_{e l}(\mu)$. The lower bounds on $\alpha_{\theta}$ were actually chosen from the numerical data rather than from the curves. 


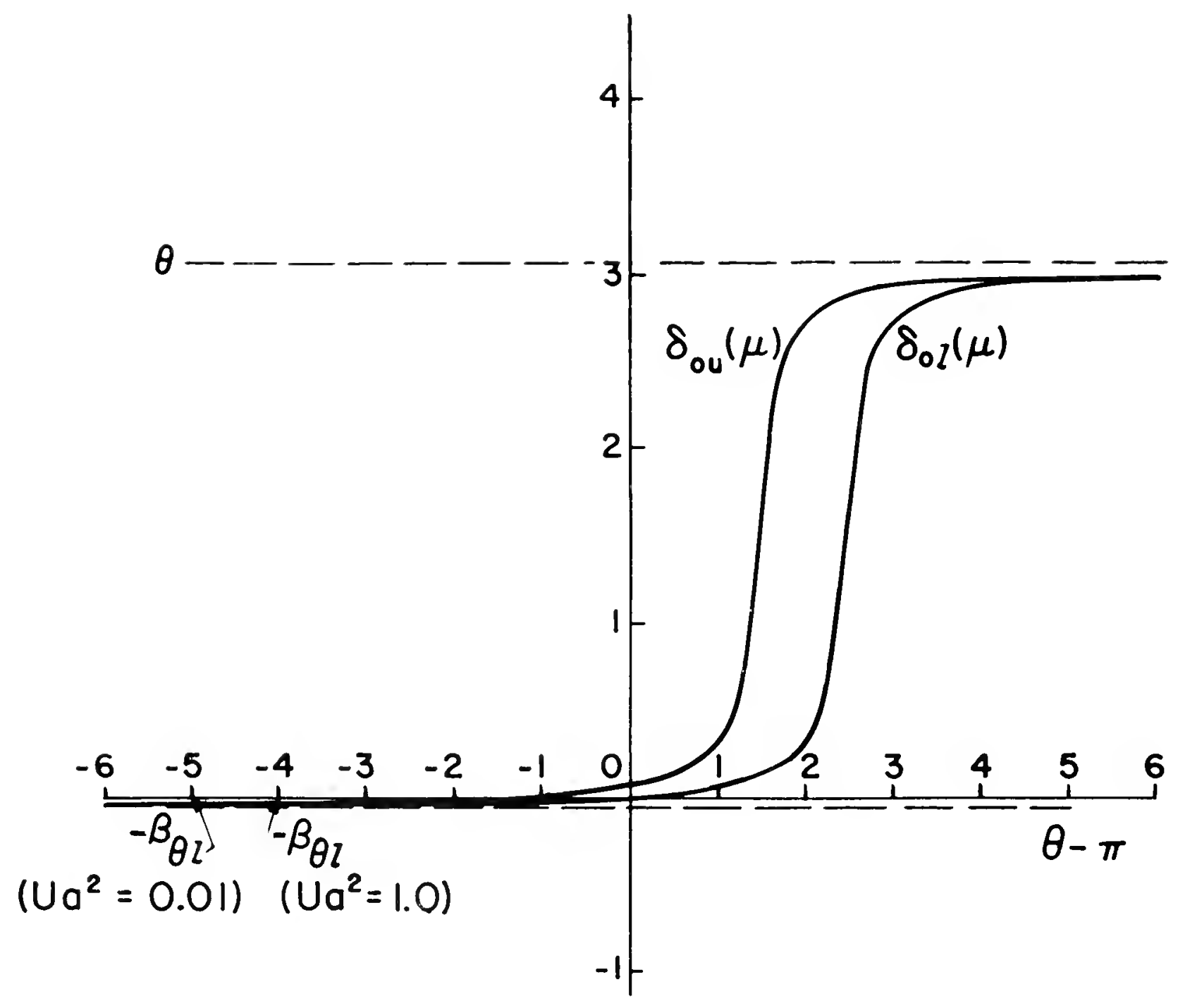

\section{Figure 4}

Upper and lower bounds on $\delta_{0}(\mu)$ for $\mathrm{ka}=0.1, \mathrm{Ua}^{2}=1.0$. The lower bound, $\delta_{\mathrm{ol}}(\mu)$, also applies to $\mathrm{ka}=0.1, \mathrm{Ua}^{2}=0.1$.

The lower bounds on $\beta_{\theta}$ were actually chosen from the numerical data rather than from the curves. Both bounds were derived from the same curve and could have been chosen equal. 


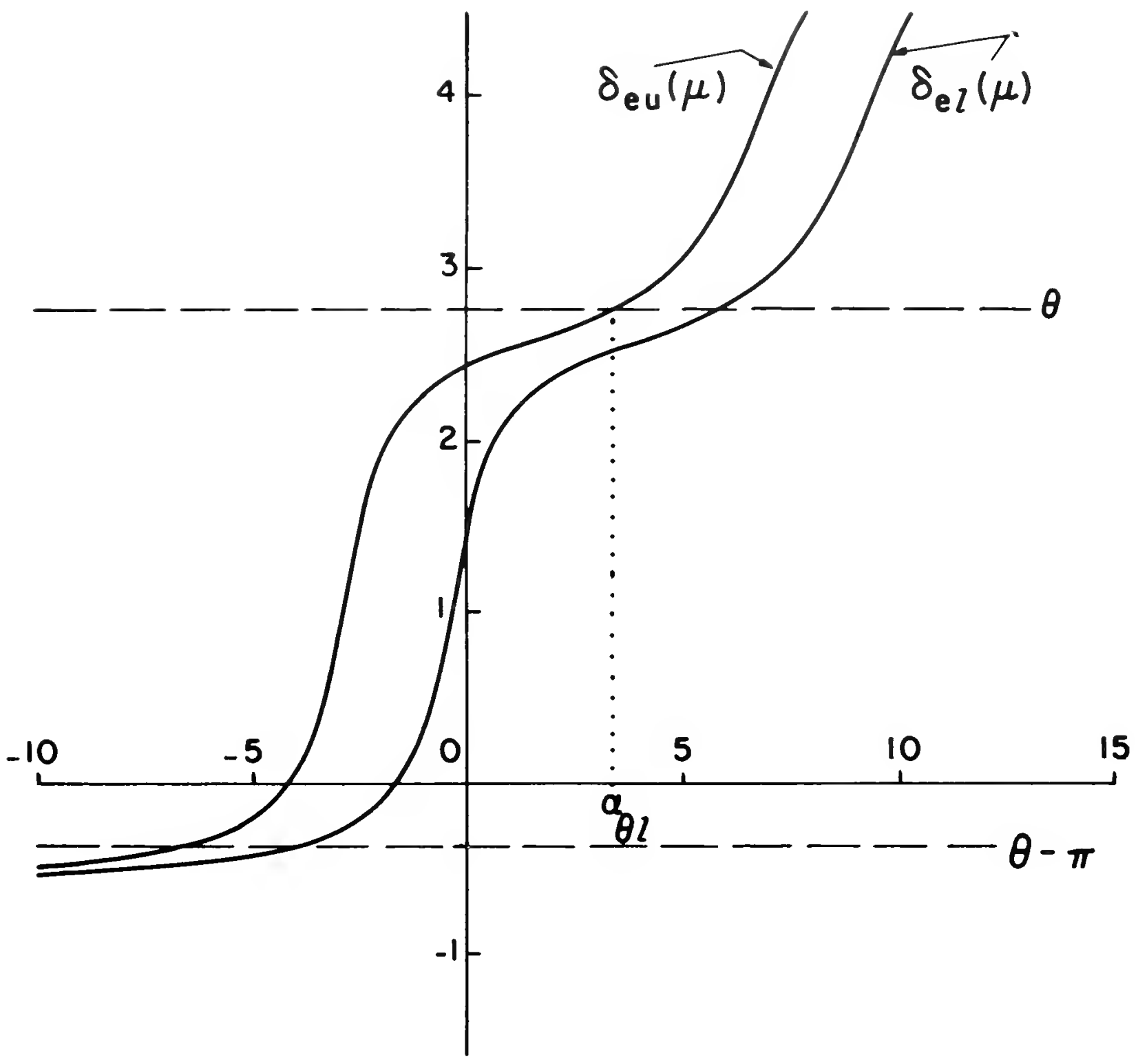

Figure 5

Upper and lower bounds on $\delta_{e}(\mu)$ for $\mathrm{ka}=\pi / 4, \mathrm{Ua}^{2}=\pi^{2} / 4$. 


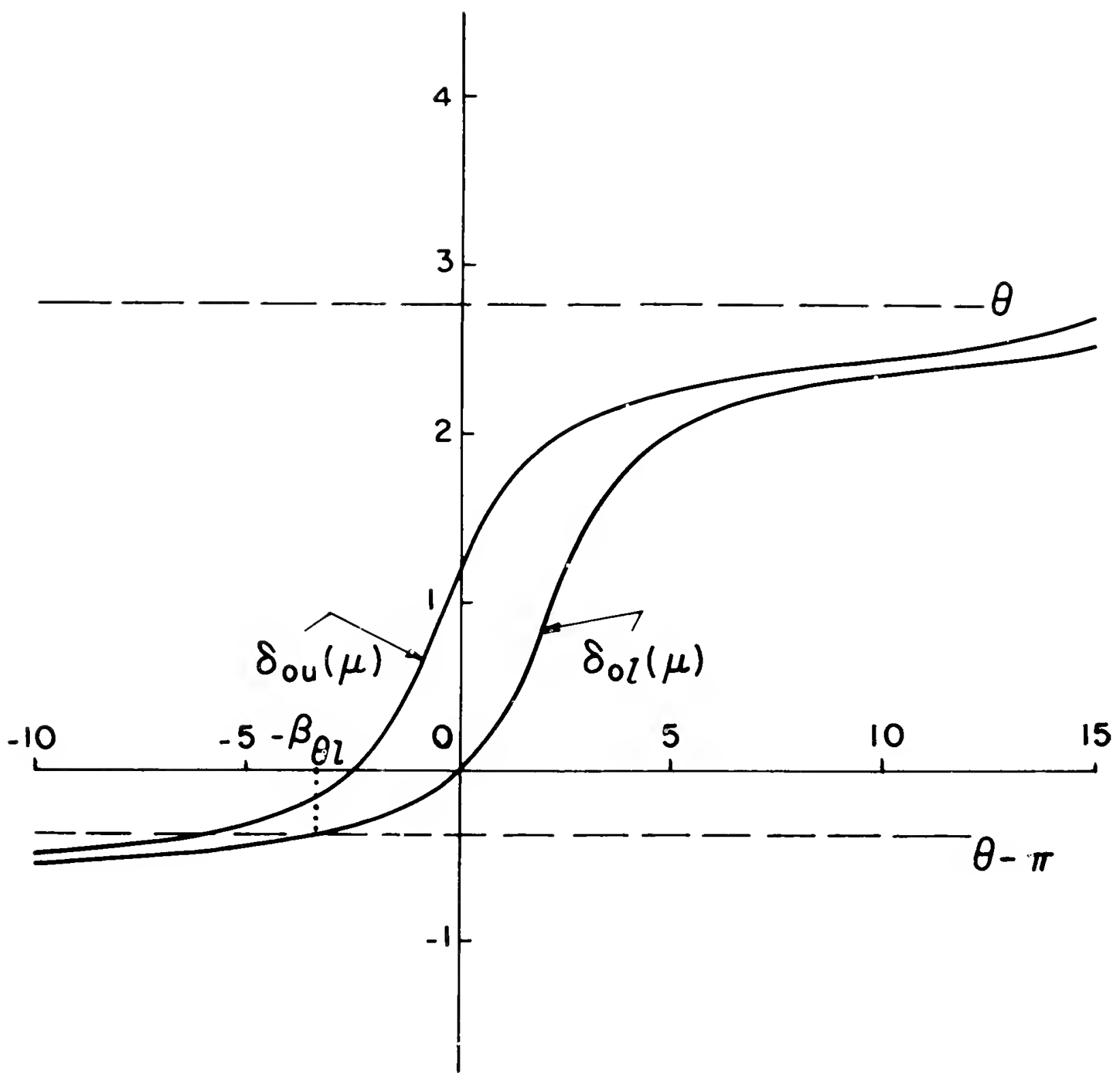

Figure 6

Upper and lower bounds on $\delta_{0}(\mu)$ for $\mathrm{ka}=\pi / 4, \mathrm{Ua}{ }^{2}=\pi^{2} / 4$. 
Table I. Values of $\theta$ and lower bounds $\alpha_{\theta l}$ and $\beta_{\theta l}$ for three choices of $\mathrm{ka}$ and $\mathrm{Ua}^{2}$, obtained from Figs. 3, 4, 5 and 6 .

\begin{tabular}{ccccc}
\hline $\mathrm{ka}$ & $\mathrm{Ua}^{2}$ & $\theta$ & $\alpha_{\theta \ell}$ & $\beta_{\theta l}$ \\
\hline 0.1 & 0.01 & 3.08359 & 5.0 & 5.0 \\
0.1 & 1.0 & 3.08359 & 4.0 & 4.0 \\
$\pi / 4$ & $\pi^{2} / 4$ & 2.775 & 3.3 & 3.3 \\
\hline
\end{tabular}




$$
e_{n \theta}=\left(\begin{array}{l}
1 \\
0
\end{array}\right),\left(\begin{array}{l}
0 \\
1
\end{array}\right) \text {, and }\left(\begin{array}{l}
1 \\
1
\end{array}\right) \text {. }
$$

The corresponding values of $e_{m} \theta_{m} B_{\theta} e^{e}$ are $B_{11}, B_{22}$, and $B_{11}+B_{22}+2 B_{12}$. While it has been noted that three different values of $e \theta$ are required in order to obtain bounds on all three independent elements of ${ }_{m} \theta$, no criterion has been given for selecting these values. It is probably desirable to bound $B_{11}$ and $B_{22}$ separately but the remaining value of $e_{m} \theta$ could, in principle, be selected to provide the closest bounds on $\mathrm{B}_{12}$. However, in the calculations presented here no attempt has been made to optimize the choice of $\mathrm{e}_{\boldsymbol{\omega}}$.

The calculations were based on the trial function given by Fq. (54) with terms up to $x^{3}$, so that there were four linear variational parameters. Due to the two boundary conditions at $\mathrm{x}=\mathrm{a}$, this corresponds to two free parameters. The free parameters were varied to give the best bounds. The bounds obtained on the various combinations of the elements of $\mathrm{B}_{\boldsymbol{m}}$ are listed in Table 2. The bounds are seen to be very much closer in the case in which $\mathrm{ka}=0.1, \mathrm{Ua}^{2}=0.01$ than in the other two cases. This result is to be expected, for considering $k$ to be fixed, this case involves a less effective potential than either of the other two. Note that for the secona and third sets of values, the potential is by no means negligible as can be seen (Table 4) from the exact values of the phase shifts and the mixing parameters; for zero potentials, one would have $\eta_{e}=90^{\circ}, \eta_{0}=0^{\circ}$, and $\psi=0^{\circ}$. 


\section{$28 a$}

Table 2. Upper and lower bounds on combinations of the elements of the susceptance matrix for three choices of $\mathrm{ka}, \mathrm{Ua}^{2}$ and $\theta$, compared with the exact values. Two parameters were used in the calculations, and lower bounds on $\alpha_{\theta}$ and $\beta_{\theta}$ were determined from Figs. 3, 4, 5 and 6 .

\begin{tabular}{|c|c|c|c|}
\hline quantity & $\begin{array}{l}\text { upper } \\
\text { bound }\end{array}$ & $\begin{array}{l}\text { lower } \\
\text { bound }\end{array}$ & $\begin{array}{l}\text { exact } \\
\text { value }\end{array}$ \\
\hline \multicolumn{4}{|c|}{$\mathrm{ka}=0.1, \mathrm{Ua}^{2}=0.01$} \\
\hline $\mathrm{B}_{11 .}$ & 0.1080314 & 0.1080083 & 0.1080210 \\
\hline$B_{22}$ & -17.1726221 & -17.1726309 & $-17 \cdot 1726230$ \\
\hline $\mathrm{B}_{11}+\mathrm{B}_{22}+2 \mathrm{~B}_{12}$ & -16.9786186 & -16.9786503 & -16.9786300 \\
\hline \multicolumn{4}{|c|}{$\mathrm{ka}=0.1, \mathrm{Ua}^{2}=1.0$} \\
\hline $\mathrm{B}_{11}$ & 4.87112 & 4.43705 & 4.71216 \\
\hline $\mathrm{B}_{22}$ & -12.95737 & -13.08907 & -12.98583 \\
\hline $\mathrm{B}_{11}+\mathrm{B}_{22}+2 \mathrm{~B}_{12}$ & -0.64432 & -1.14168 & -0.85319 \\
\hline \multicolumn{4}{|c|}{$\mathrm{ka}=\pi / 4, \mathrm{Ua}^{2}=\pi^{2} / 4$} \\
\hline $\mathrm{B}_{11}$ & 1.76488 & 1.23977 & 1.58228 \\
\hline $\mathrm{B}_{22}$ & -1.22869 & -1.31261 & -1.25519 \\
\hline $\mathrm{B}_{11}+\mathrm{B}_{22}+2 \mathrm{~B}_{12}$ & 2.32112 & 1.75493 & 2.09091 \\
\hline
\end{tabular}


Table 3. Bounds on $B_{11}$ for $\mathrm{ka}=\pi / 4, \mathrm{Ua}^{2}=\pi^{2} / 4$ for aifferent numbers of parameters. The values of $\theta, \alpha_{\theta \ell}$ and $\beta_{\theta l}$ are as shown in Table 1.

\begin{tabular}{cccc}
\hline $\begin{array}{c}\text { number of free } \\
\text { parameters }\end{array}$ & $\begin{array}{c}\text { upper } \\
\text { bound }\end{array}$ & $\begin{array}{c}\text { lower } \\
\text { bound }\end{array}$ & $\begin{array}{c}\text { exact } \\
\text { value }\end{array}$ \\
\hline 2 & 1.76488 & 1.23977 & 1.58228 \\
4 & 1.69009 & 1.45289 & 1.58228 \\
6 & 1.65803 & 1.49871 & 1.58228 \\
\hline
\end{tabular}




\section{$28 c$}

Table 4. Upper and lower bounds on the eigenphase shifts and mixing parameters for three choices of $\mathrm{ka}, \mathrm{Ua}^{2}$ and $\theta$, compared with the exact values. These quantities were calculated from the bounds on the combinations of the elements of $\underset{m}{B}$ listed in Table 2, using Eqs. (62).

\begin{tabular}{|c|c|c|c|}
\hline quantity & $\begin{array}{l}\text { upper } \\
\text { bound }\end{array}$ & $\begin{array}{l}\text { lower } \\
\text { bound }\end{array}$ & $\begin{array}{l}\text { exact } \\
\text { value }\end{array}$ \\
\hline \multicolumn{4}{|l|}{$\mathrm{ka}=0.1, \mathrm{Ua}^{2}=0.01$} \\
\hline$\psi$ & $-0^{\circ} 8132.90^{\prime \prime}$ & $-0^{\circ} 8: 33.29^{\prime \prime}$ & $-0^{\circ} 8133.08^{\prime \prime}$ \\
\hline$\eta_{e}$ & $92^{\circ} 50^{\prime} 56^{\prime \prime}$ & $92^{\circ} 50^{\prime} 50^{\prime \prime}$ & $92^{\circ} 50^{\prime} 53^{\prime \prime}$ \\
\hline$\eta_{0}$ & $0^{\circ} 0^{1} 34.28^{\prime \prime}$ & $0^{\circ} 0^{\prime} 34.24 "$ & $0^{\circ} 0^{\prime} 34.26^{\prime \prime}$ \\
\hline \multicolumn{4}{|l|}{$\mathrm{ka}=0.1, \mathrm{Ua}^{2}=1.0$} \\
\hline$\psi$ & $-10^{\circ} 34^{\prime}$ & $-12^{\circ} 16^{\prime}$ & $-11^{\circ} 23^{\prime}$ \\
\hline$\eta_{e}$ & $167^{\circ} 12^{\prime}$ & $165^{\circ} 7^{\prime}$ & $166^{\circ} 18^{\prime}$ \\
\hline$\eta_{0}$ & $1^{\circ} 0^{\prime}$ & $0^{0} 4^{\prime}$ & $0^{\circ} 51^{\prime}$ \\
\hline \multicolumn{4}{|l|}{$\mathrm{ka}=\pi / 4, \mathrm{Ua}^{2}=\pi^{2} / 4$} \\
\hline$\psi$ & $-10^{\circ} 48^{\prime}$ & $-22^{0} 4^{\prime}$ & $-15^{\circ} 56^{\prime}$ \\
\hline$\eta_{e}$ & $137^{\circ} 27^{\prime}$ & $116^{\circ} 3^{\prime}$ & $130^{\circ} 24^{\prime}$ \\
\hline$\eta_{0}$ & $27^{\circ} 33^{\prime}$ & $1^{\circ} 30^{\prime}$ & $11^{0} 34^{r}$ \\
\hline
\end{tabular}


The effect of increasing the number of parameters is shown in Table 3, which shows bounds on $\mathrm{B}_{11}$ for the case $\mathrm{ka}=\pi / 4, \mathrm{Ua}^{2}=\pi^{2} / 4$, calculated with 2, 4, and 6 free parameters. While the bounds improve as the number of free parameters is increased, the improvement is not as rapid as one might expect. This result is attributed to the poor choice of the form of the trial function. The trial function used in calculating upper bounds on $B_{11}$ with 2 parameters is compared with the exact function in Figure 7 .

Finally, bounds on the eigenphase shifts and mixing parameters are listed in Table 4. These quantities were calculated from the bounds on combinations of the elements of $\mathrm{B}_{\theta}$ listed in Table 2, using Eqs. (52). It would be a trivial matter to also obtain bounds on the elements of $\mathrm{S}$, but the title of the paper notwithstanding, we have not bothered to do so.

From the foregoing results, it is clear that useful bounds can be calculated on the independent quantities which determine the scattering matrix in a multi-channel problem, even with relatively extreme values for the depth and range of the potential. Furthermore, since diagonal comparison potentials can be used, the calculation requires no more than the exact solution of related single-channel problems. Thus it is anticipated that the method presented here can provide useful results in many multi-channel problems for which there would otherwise be no practical approach.

\section{ACKNOWLEDGMENTS}

Special thanks are due to Dr. Martin Kelly and Dr. Leonard Rosenberg for a number of helpful discussions. 


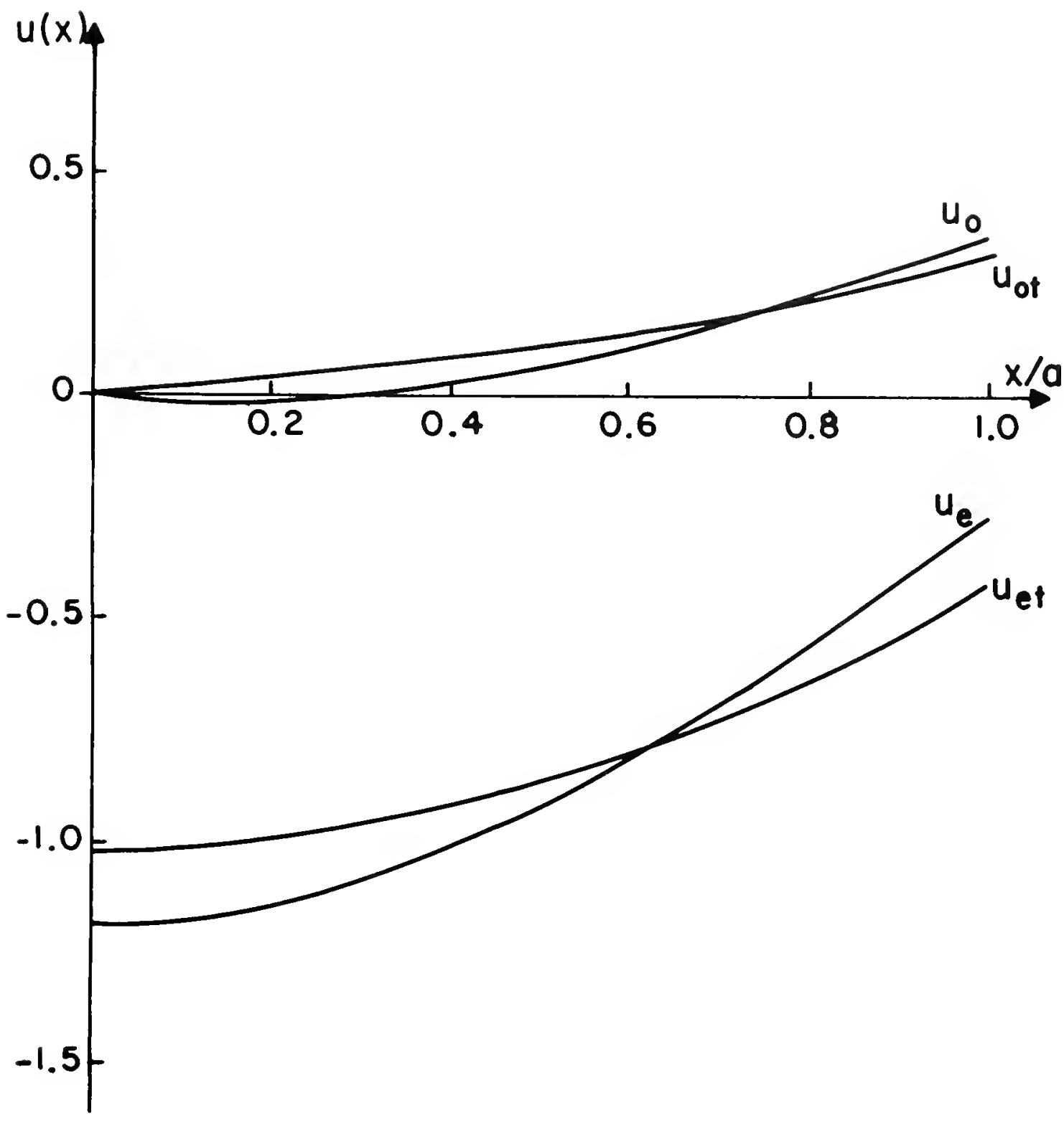

Figure 7

Comparison of trial function with exact function. The trial function, which contains two free parameters, was used to calculate an upper bound on $B_{11}$ for the case in which $k a=\pi / 4$, $\mathrm{Ua}^{2}=\pi^{2} / 4$. 


\section{REFERENCES}

$I_{\text {T. Kato, Progr. Theoret. Phys. (Kyoto) }}$ m, 394 (1951).

2 L. Spruch and M. Kelly, Phys. Rev. 109, 2144 (1958).

3. Spruch, Phys. Rev. 109, 2149 (1958).

${ }^{4}$ L. Spruch and I. Rosenberg, Phys. Rev. 116, 1034 (1959).

${ }^{5}$ L. Spruch and R. Bartram, J.A.P. 31,905 (1960); R. Bartram and L. Spruch, J.A.P. 31, $913(1960)$.

6.M. Blatt and V.F. Weisskopf, Theoretical Nuclear Physics (John Wiley and Sons, Inc., New York, 1952) p. 102.

7 op. cit., p. 530

8. Spruch and L. Rosenberg, Phys. Rev. 116, 1034 (1959); 117 , 1095 (1960); L. Rosenberg, L. Spruch and T.F. O'Malley, Phys. Rev. II8, I84 (1960); L. Rosenberg and L. Spruch, Phys. Rev. 120, 474 (1960); $\lim _{\lim } 1720(1961)$.

${ }^{9}$ For incident energies greater than zero, the various potentials have to be truncated, but this will probably not be a serious limitation in most applications. We might note in passing that the papers referred to actually provide a method for determining $\beta$, at least for truncated potentials, but we have just noted that this is no longer particularly interesting.

${ }^{10}$ L. Rosenberg and I. Spruch, to be published.

llMatrix quantities will be denoted by boldface type. Other symbols employed are + for hermitian adjoint, $\sim$ for transpose, and * for complex conjugate. 
12 Blatt and Weisskopf, ref. 6, pp. 525, 529.

${ }^{13}$ P.M. Morse and H. Feshbach, Methods of Theoretical Physics (McGrawHill Book Company, Inc., New York, 1953).

${ }^{14}$ For a discussion of eigenphase shifts and mixing parameters, see for example W. Kohn, Phys. Rev. 74, 1763 (1948); J.M. Blatt and I.C. Biedenharn, Phys. Rev. 86, 399 (1952); I.C. Biedenharn and J.M. Blatt, Phys. Rev. 93, 1387 (1954). 


NEW YORK UNIVERSITY

INSTITUTIE OF MATHEMATICAL SCIENCES

LIBRARY

4 Washington Place, New York 3, N. Y.

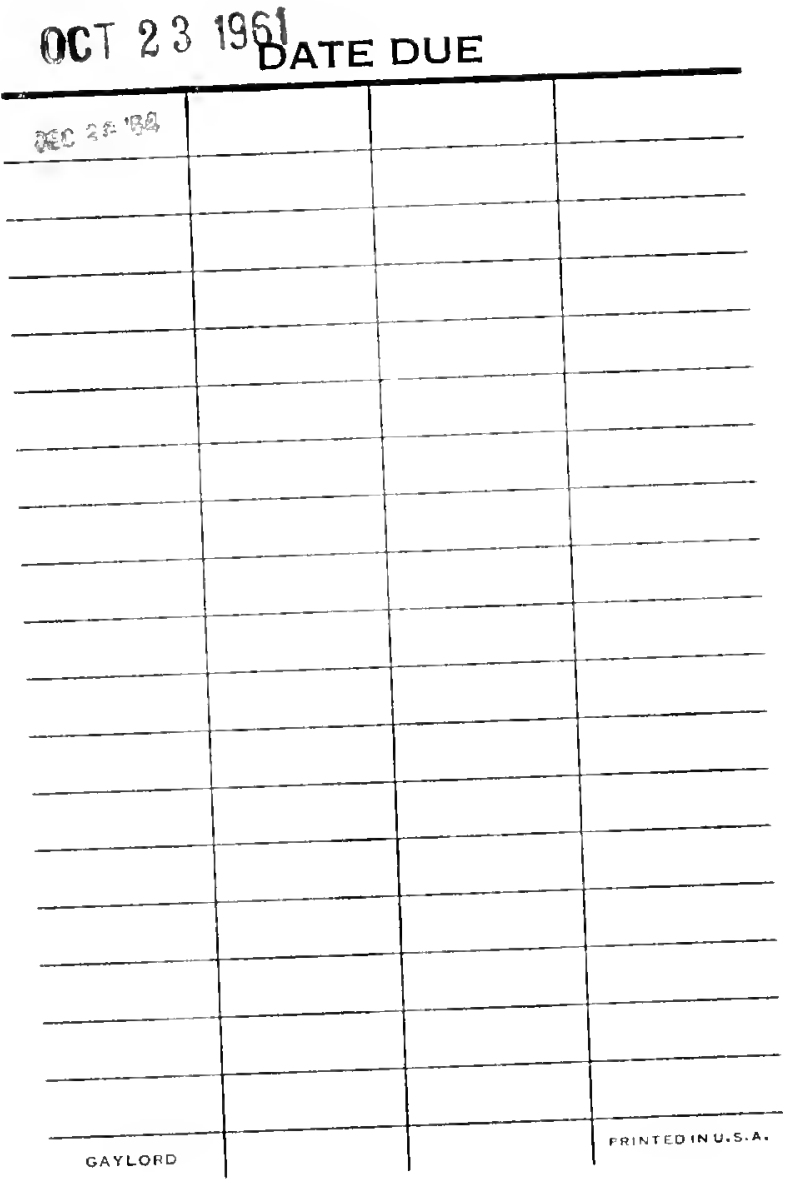




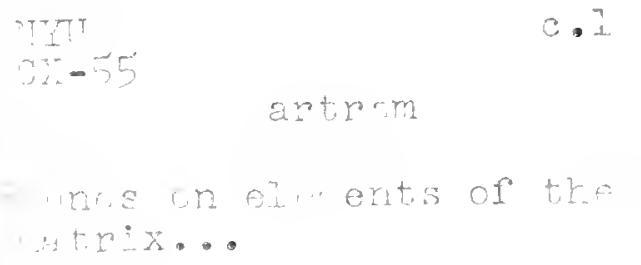

N. Y. U. Institute of Mathematical Sciences 25 Waverly Place New York 3, N. Y. 
1

3 